Supporting Information

\title{
Shear strength and interfacial toughness characterization of sapphire-epoxy interfaces for nacre-inspired composites
}

Sebastian Behr ${ }^{1}$, Laura Jungblut ${ }^{1}$, Michael V. Swain ${ }^{2,3}$, Gerold A. Schneider $^{1 *}$

${ }^{1}$ Hamburg University of Technology, Institute of Advanced Ceramics, Hamburg, Germany

${ }^{2}$ University of Sydney, Biomaterials, Faculty of Dentistry, Sydney, Australia

${ }^{3}$ Kuwait University, Bioclinical Sciences, Faculty of Dentistry, Kuwait City, Kuwait

* E-mail: g.schneider@tuhh.de 
Supporting Information - Behr et al. Shear strength and interfacial toughness characterization [...]

\section{GENERAL COMMENTS}

Data presented here has been prepared with utmost care. Nevertheless, errors cannot be fully excluded. Reference numbers correspond to the bibliography of the main article that also defines the meanings of symbols, quantities, and abbreviations used here.

\section{INTERFACIAL SHEAR STRENGTH FROM SCHWICKERATH TESTS}

The interfacial shear strength is determined according to Lenz et al. ${ }^{29}$ and DIN EN ISO $9693^{28}$ and the interested reader is referred to these publications for further details. Here, it shall just be stated that the interfacial shear strength $\tau_{\mathrm{i} \text {, max }}$ can be derived from the peak force $F_{\text {peak }}$, the substrate modulus $E_{1}$ and the substrate thickness $t$ following

$$
\begin{aligned}
& \tau_{\mathrm{i}, \max }=k\left(t, E_{1}\right) \cdot F_{\text {peak }} \quad \text { with } \\
& k\left(t, E_{1}\right)=A\left(E_{1}\right) \cdot t^{2}+B\left(E_{1}\right) \cdot t+C\left(E_{1}\right) .
\end{aligned}
$$

Calculations are quite complex as shown in Figure S1 and literature ${ }^{28,29}$. They are based, however, on a general fit to finite element simulation data. The parameters $\mathrm{A}, \mathrm{B}$, and $\mathrm{C}$ from the polynomial in equation (S1) are functions of the actual substrate modulus. For the one determined for c-sapphire in this work, $432 \mathrm{GPa}$, they amount to

$$
A \approx 5.342 \mathrm{~mm}^{-4}, B \approx-11.353 \mathrm{~mm}^{-3} \text {, and } C \approx 7.065 \mathrm{~mm}^{-2}
$$

With these, the average substrate thickness $t=0.54 \mathrm{~mm}$ stated in the main article leads to $k=2.49 \mathrm{~mm}^{-2}$. Detailed data for every individual sample is listed in Table S1.

\section{INTERFACIAL SHEAR TOUGHNESS FROM SCHWICKERATH TESTS}

The foundation for the toughness analysis is given by Schneider \& Swain for brittle joints with ideal interfacial bonding. As demonstrated in the main article, this theory is similarly 
Supporting Information - Behr et al. Shear strength and interfacial toughness characterization $[\ldots]$

applicable to non-ideal bondings and inelastically deformable interfaces, if two main adaptions are made: (i) The product $E I$ has to be determined from the experiments and cannot be calculated theoretically and (ii) every data point that is considered for toughness analysis needs a parallel shift by the inelastic deformation that accumulated up to this point to accurately determine the toughness for this crack length. Both measures are post-mortem adjustments.

(i) The product EI can be calculated from and directly affects the crack-length-dependent compliance $C(a)$ of every sample, ${ }^{31}$

$$
\begin{aligned}
& C(a)=\frac{1}{12}\left[\frac{[l-b]^{3}+[l-b+a]^{3}}{E_{1} I_{10}}+\frac{2 l^{3}-[l-b]^{3}-[l-b+a]^{3}}{E I}\right] \quad \forall a \leq b, \\
& C(a)=\frac{1}{12}\left[\frac{2 l^{3}+[l-b]^{3}-[l+b-a]^{3}}{E_{1} I_{10}}+\frac{[l+b-a]^{3}-[l-b]^{3}}{E I}\right] \quad \forall a>b .
\end{aligned}
$$

The subsequent procedure to obtain the theoretical toughness maps from this in-situ determination of the compliance is explained in the main article.

(ii) The procedure to obtain a data point for the $R$-curve analysis starts with selecting a point with a known crack length $a$. The latter is achieved by comparing the compliance of an unloading curve with the theoretical compliance for various crack length according to equations (S1) and (S2). Extrapolation of the unloading curve to the horizontal axis at zero force provides the apparent inelastic deformation. A parallel shift of the data point under consideration by this apparent inelastic deformation finally ends inside the toughness map and on a curve of constant toughness. Its value is considered as the toughness related to the crack length associated with the shifted data point. The latter is reasonable since cracks are driven by small increments. Consequently, the determined crack length is almost identical to its preceding one that effectively belongs to the toughness obtained above. The parallel shift of the data point is similar to a parallel expansion of the underlying toughness map in the opposite direction und yields 
Supporting Information - Behr et al. Shear strength and interfacial toughness characterization [...]

similar results but is much more convenient to read. Anyway, it has to be kept in mind that it remains unknown whether the apparent inelastic deformation is indeed contributing to any toughening of the interface.

\section{MODELS TO PREDICT THE STRENGTH OF NACRE-INSPIRED COMPOSITES}

Several models have been proposed to predict the strength $\sigma_{\mathrm{c}}$ of planar-reinforced (nacreinspired) composites, amongst others by Padawer \& Beecher ${ }^{16}$ and Jackson et al. ${ }^{42}$ Generally, these composites can fail in two major ways, by particle fracture or by particle pull-out. Naturally, the former is governed by the tensile strength of the particles, $\sigma_{\mathrm{p}}$, and the stress transfer through the interface and from the surrounding matrix. Particle pull-out on the other hand may happen after matrix yielding and matrix failure (cohesive failure) in case of a strong interfacial bonding or by simple interfacial failure in case of a poor bonding. Hence, either the matrix shear strength $\tau_{\mathrm{m}}$ or the interfacial shear strength $\tau_{\text {if }}$ are reached first. To comply with the models listed above, an interphase shear strength $\tau_{\text {ip }}=\min \left[\tau_{\mathrm{m}} ; \tau_{\text {if }}\right]$ shall be defined here. The stress transfer and stress distribution between matrix and particles generally depends on the particle volume fraction $v$, Young's modulus of particles and matrix, $E_{\mathrm{p}}$ and $E_{\mathrm{m}}$, the shear modulus $G_{\mathrm{m}}$ of the matrix, and the aspect ratio $\alpha$ of the particles, the ratio between length and thickness of the particles. Finally, there is a critical aspect ratio that estimates the transition between particle pull-out (small aspect ratio) and particle fracture (large aspect ratio) by means of an incomplete or a complete stress transfer from the matrix into the particles.

Generally, this critical aspect ratio is approximated as $\alpha_{\mathrm{c}} \approx \sigma_{\mathrm{p}} / \tau_{\mathrm{m}} \cdot{ }^{2,4,16,42,43}$

With this nomenclature, the models by Padawer \& Beecher ${ }^{16}$ and Jackson et al. ${ }^{42}$, as examples, now lead to the following mathematical expressions: 
Supporting Information - Behr et al. Shear strength and interfacial toughness characterization [...]

Padawer \& Beecher: $\sigma_{\mathrm{c}} \leq \begin{cases}v \cdot \sigma_{\mathrm{p}} \cdot \frac{1-\frac{\tanh [u]}{u}}{1-\operatorname{sech}[u]} & \forall \alpha \geq \alpha_{\mathrm{c}} \\ v \cdot \tau_{\mathrm{ip}} \cdot \frac{\alpha}{u} \cdot\left(\frac{1}{\tanh [u]}-\frac{1}{u}\right) & \forall \alpha<\alpha_{\mathrm{c}}\end{cases}$

Jackson et al.: $\quad \sigma_{\mathrm{c}} \leq \begin{cases}v \cdot \sigma_{\mathrm{p}} \cdot\left[1-\frac{\alpha_{\mathrm{c}}}{2 \alpha}\right] & \forall \alpha \geq \alpha_{\mathrm{c}} \\ \frac{1}{2} v \cdot \tau_{\mathrm{ip}} \cdot \alpha & \forall \alpha<\alpha_{\mathrm{c}}\end{cases}$

Consequently, the composite strength linearly depends on the interphase shear strength and thus on the interfacial bonding, if the latter is relatively poor or the aspect ratio small.

Therefore, a manifold increase in the interfacial shear strength could lead to the same increase in composite strength. Hence, tailored particle-matrix interfaces are essential for advanced nacre-inspired composites. 


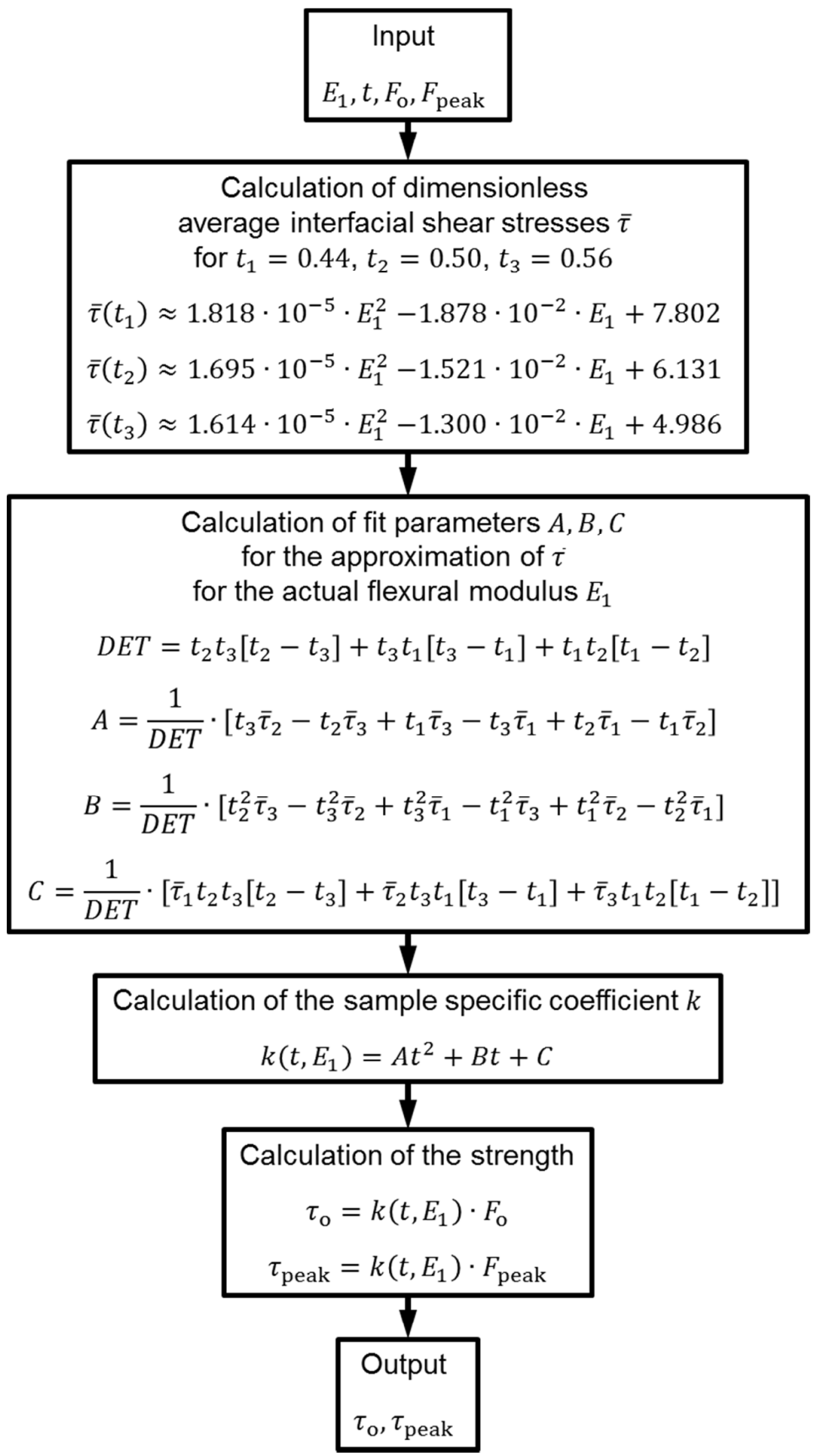

Figure S1. Flow chart of the algorithm used for determination of the interfacial shear strength. It is an adaption of the ones published in DIN EN ISO $9693^{28}$ and by Lenz et al. ${ }^{29}$ 
Supporting Information - Behr et al. Shear strength and interfacial toughness characterization [...]

Table S1. Data sets for the determination of the interfacial shear strength.

\begin{tabular}{|c|c|c|c|c|c|c|c|}
\hline \multirow{3}{*}{$\begin{array}{l} \\
E_{1} \\
{[\mathrm{GPa}]}\end{array}$} & Sample 1 & Sample 2 & Sample 3 & Sample 4 & Sample 5 & Average & StD \\
\hline & \multicolumn{7}{|c|}{ as-received sapphire } \\
\hline & 432 & 432 & 432 & 432 & 432 & 432 & - \\
\hline $\begin{array}{l}t \\
{[\mathrm{~mm}]}\end{array}$ & 0.535 & 0.543 & 0.535 & 0.530 & 0.540 & 0.537 & 0.005 \\
\hline $\begin{array}{l}F_{\mathrm{o}} \\
{[\mathrm{N}]}\end{array}$ & 2.49 & 3.23 & 3.55 & 3.03 & 3.78 & 3.22 & 0.50 \\
\hline $\begin{array}{l}F_{\text {peak }} \\
{[\mathrm{N}]}\end{array}$ & 3.59 & n.a. & 4.64 & 3.93 & 4.84 & 4.25 & 0.59 \\
\hline $\begin{array}{l}k \\
{\left[\mathrm{~mm}^{-2}\right]}\end{array}$ & 2.520 & 2.475 & 2.520 & 2.548 & 2.492 & 2.511 & 0.028 \\
\hline $\begin{array}{l}\tau_{\mathrm{o}} \\
{[\mathrm{MPa}]}\end{array}$ & 6.28 & 8.00 & 8.95 & 7.72 & 9.42 & 8.07 & 1.22 \\
\hline \multirow[t]{2}{*}{$\begin{array}{l}\tau_{\mathrm{i}, \max } \\
{[\mathrm{MPa}]}\end{array}$} & 9.05 & n.a. & 11.69 & 10.02 & 12.06 & 10.70 & 1.42 \\
\hline & \multicolumn{7}{|c|}{ thermally modified sapphire } \\
\hline $\begin{array}{l}E_{1} \\
{[\mathrm{GPa}]}\end{array}$ & 432 & 432 & 432 & 432 & 432 & 432 & \\
\hline $\begin{array}{l}t \\
{[\mathrm{~mm}]}\end{array}$ & 0.548 & 0.532 & 0.537 & 0.544 & 0.532 & 0.539 & 0.007 \\
\hline $\begin{array}{l}F_{\mathrm{o}} \\
{[\mathrm{N}]}\end{array}$ & 13 & 11.77 & 11.25 & 11.81 & 10.89 & 11.74 & 0.80 \\
\hline $\begin{array}{l}F_{\text {peak }} \\
{[\mathrm{N}]}\end{array}$ & 14.52 & 12.92 & 13.58 & 12.97 & 14.28 & 13.65 & 0.73 \\
\hline $\begin{array}{l}k \\
{\left[\mathrm{~mm}^{-2}\right]}\end{array}$ & 2.448 & 2.537 & 2.509 & 2.470 & 2.537 & 2.500 & 0.040 \\
\hline $\begin{array}{l}\tau_{\mathrm{o}} \\
{[\mathrm{MPa}]}\end{array}$ & 31.82 & 29.86 & 28.23 & 29.17 & 27.22 & 29.26 & 1.74 \\
\hline $\begin{array}{l}\tau_{\mathrm{i}, \max } \\
{[\mathrm{MPa}]}\end{array}$ & 35.54 & 32.78 & 34.07 & 32.03 & 36.23 & 34.13 & 1.78 \\
\hline
\end{tabular}

